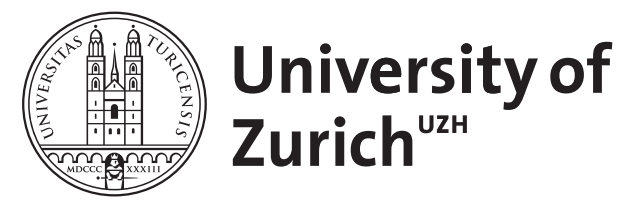

\title{
Trees and ultrametric Möbius structures
}

\author{
Beyrer, Jonas ; Schroeder, Victor
}

\begin{abstract}
We define the concept of an ultrametric Möbius space $(\mathrm{Z}, \mathrm{M})$ and show that the boundary at infinity of a nonelementary geodesically complete tree is naturally an ultrametric Möbius space. In addition, we construct to a given ultrametric Möbius space $(\mathrm{Z}, \mathrm{M})$ a nonelementary geodesically complete tree, unique up to isometry, with $(\mathrm{Z}, \mathrm{M})$ being its boundary at infinity. This yields a one-to-one correspondence.
\end{abstract}

DOI: https://doi.org/10.1134/S207004661704001X

Posted at the Zurich Open Repository and Archive, University of Zurich

ZORA URL: https://doi.org/10.5167/uzh-142991

Journal Article

Accepted Version

Originally published at:

Beyrer, Jonas; Schroeder, Victor (2017). Trees and ultrametric Möbius structures. p-Adic Numbers, Ultrametric Analysis and Applications, 9(4):247-256.

DOI: https://doi.org/10.1134/S207004661704001X 


\title{
Trees and ultrametric Möbius structures
}

\author{
Jonas Beyrer \& Viktor Schroeder*
}

\begin{abstract}
We define the concept of an ultrametric Möbius space and use this to characterize nonelementary geodesically complete trees.
\end{abstract}

Keywords Trees, Möbius structures, ultrametrics

Mathematics Subject Classification 53C35, 53C23

\section{Introduction}

In this paper we define the concept of an ultrametric Möbius space $(Z, \mathcal{M})$, where $Z$ is some set with cardinality $|Z| \geq 3$ and $\mathcal{M}$ an ultrametric Möbius structure (see section 2). These Möbius spaces describe in a natural way the asymptotic geometry of trees. To a metric tree $(X, d)$ one can associate in a natural way a boundary at infinity $\partial_{\infty} X$. We call a tree $(X, d)$ geodesically complete, if every geodesic segment can be extended to a complete geodesic line. We call a tree nonelementary, if $\left|\partial_{\infty} X\right| \geq 3$. The boundary $\partial_{\infty} X$ of a nonelementary tree $(X, d)$ carries a canonical ultrametric Möbius structure $\mathcal{M}_{X}$. If $F:(X, d) \rightarrow\left(X^{\prime}, d^{\prime}\right)$ is an isometric embedding of trees, then $F$ extends in a natural way to a Möbius map $f:\left(\partial_{\infty} X, \mathcal{M}_{X}\right) \rightarrow\left(\partial_{\infty} X, \mathcal{M}_{X^{\prime}}\right)$.

We show that the association $(X, d) \mapsto\left(\partial_{\infty} X, \mathcal{M}_{X}\right)$ defines an equivalence between the category of isometry classes of nonelementary geodesically complete trees and the category of Möbius classes of ultrametric Möbius spaces. Therefore we will prove the following facts:

1. Let $(Z, \mathcal{M})$ be a complete ultrametric Möbius space. Then there exists a unique nonelementary geodesically complete tree $(X, d)$, such that $\left(\partial_{\infty} X, \mathcal{M}_{X}\right)$ is Möbius equivalent to $(Z, \mathcal{M})$.

2. Let $(X, d)$ and $\left(X^{\prime}, d^{\prime}\right)$ be nonelementary geodesically complete trees. Then for any Möbius embedding $f:\left(\partial_{\infty} X, \mathcal{M}_{X}\right) \rightarrow\left(\partial_{\infty} X^{\prime}, \mathcal{M}_{X^{\prime}}\right)$, there exists a unique isometric embedding $F:(X, d) \rightarrow\left(X^{\prime}, d^{\prime}\right)$ such that the extension of $F$ to the boundary coincides with $f$. If $f$ is in addition surjective, then $F$ is an isometry.

\footnotetext{
${ }^{*}$ Supported by Swiss National Science Foundation Grant 153607
} 
These results are in the spirit of [Hu], who investigated the case of trees with a basepoint. Probably all of the results of this paper are known in some way but usually formulated in a different language. To our opinion the viewpoint using ultrametric Möbius structures is new and worth to be formulated. Our paper is also inspired by the work of [Bi]. In this paper the author associates to a certain Möbius space $(Z, \mathcal{M})$ a filling $(X, d)$ in the more general context of $\operatorname{CAT}(-1)$ spaces. The idea in $[\mathrm{Bi}]$ is to define the filling as the subset $\mathcal{M}_{1}^{a} \subset \mathcal{M}_{X}$ of antipodal diameter 1 metrics in $\mathcal{M}_{X}$ with a canonical metric $d_{\mathcal{M}_{1}^{a}}$ on it (compare section 5). Indeed one obtains the following fact (which is a slight generalization of a result in $[\mathrm{Bi}]$ ):

3. Let $(Z, \mathcal{M})$ be a complete ultrametric Möbius space, $\mathcal{M}_{1}^{a} \subset \mathcal{M}_{X}$ the subset of antipodal diameter 1 metrics and $(X, d)$ the tree from fact 1. Then $i:(X, d) \rightarrow\left(\mathcal{M}_{1}^{a}, d_{\mathcal{M}_{1}^{a}}\right), x \mapsto \rho_{x}$ is an isometry. Here $\rho_{x}$ is the Bourdon metric at $x$.

The structure of the paper is as follows. In section 2 we introduce the concept of ultrametric Möbius spaces, in section 3 we recall some facts about metric trees. In section 4 we prove facts 1 and 2 and in the final section 5 we show fact 3 .

\section{Ultrametric Möbius spaces}

\subsection{Möbius Structure}

Let $Z$ be a set with cardinality $|Z| \geq 3$. An extended metric on $Z$ is a map $\rho: Z \times Z \rightarrow[0, \infty]$, such that there exists a set $\Omega(\rho) \subset Z$ with cardinality $|\Omega(d)| \in\{0,1\}$, such that $\rho$ restricted to the set $Z \backslash \Omega(\rho)$ is a metric (taking only values in $[0, \infty))$ and such that $\rho(z, \omega)=\infty$ for all $z \in Z \backslash \Omega(\rho)$, $\omega \in \Omega(d)$. Furthermore $d(\omega, \omega)=0$.

If $\Omega(\rho)$ is not empty, we sometimes denote $\omega \in \Omega(\rho)$ simply as $\infty$ and call it the (infinitely) remote point of $(Z, \rho)$. We often write also $\{\omega\}$ for the set $\Omega(\rho)$ and $Z_{\omega}$ for the set $Z \backslash\{\omega\}$.

We consider on $(Z, \rho)$ the topology with the basis consisting of all open distance balls $B_{r}(z)$ around points in $z \in Z_{\omega}$ and the complements $D^{C}$ of all closed distance balls $D=\bar{B}_{r}(z)$. Note that $(Z, \rho)$ is a Hausdorff space.

We call an extended metric space complete, if first every Cauchy-sequence in $Z_{\omega}$ converges and secondly if the infinitely remote point $\omega$ exists in case that $Z_{\omega}$ is unbounded. For example the real line $(\mathbb{R}, \rho)$, with its standard metric is not complete as an extended metric space, while $(\mathbb{R} \cup\{\infty\}, \rho)$ is complete.

One can also characterize completeness using the following notation. 
Definition 2.1. Let $(Z, \rho)$ be an extended metric space. A sequence $\left(z_{i}\right) \subset$ $Z$ is a Cauchy-sequence in $(Z, \rho)$, if one the following is true:

1. For an arbitrary point $o \in Z_{\omega}$ there exists $C>0$ and $n \in \mathbb{N}$, such that $\rho\left(o, z_{i}\right) \leq C$ for all $i \geq n$ and the sequence $\left(z_{i}\right)_{i \geq n}$ is a Cauchy-sequence in $Z_{\omega}$ in the classical sense.

2. For an arbitrary point $o \in Z_{\omega}$ and every $C \in \mathbb{R}$, there is an $n \in \mathbb{N}$, such that $\rho\left(o, z_{i}\right) \geq C$ for all $i \geq n$.

Then completeness of $(Z, \rho)$ is equivalent to the convergence of Cauchysequences. A Cauchy-sequence of the second type is converging to the remote point $\omega$.

We say that a quadruple $(x, y, z, w) \in Z^{4}$ is admissible, if no entry occurs three or four times in the quadruple. We denote with $Q \subset Z^{4}$ the set of admissible quadruples. We define the cross ratio triple as the map crt : $Q \rightarrow$ $\Sigma \subset \mathbb{R} P^{2}$ which maps admissible quadruples to points in the real projective plane defined by

$$
\operatorname{crt}_{\rho}(x, y, z, w)=(\rho(x, y) \rho(z, w): \rho(x, z) \rho(y, w): \rho(x, w) \rho(y, z)),
$$

here $\Sigma$ is the subset of points $(a: b: c) \in \mathbb{R} P^{2}$, where all entries $a, b, c$ are nonnegative or all entries are non-positive. Note that $\Sigma$ can be identified with the standard 2-simplex, $\{(a, b, c) \mid a, b, c \geq 0, a+b+c=1\}$.

We use the standard conventions for the calculation with $\infty$. If $\infty$ occurs once in $Q$, say $w=\infty$, then $\operatorname{crt}_{\rho}(x, y, z, \infty)=(\rho(x, y): \rho(x, z): \rho(y, z))$. If $\infty$ occurs twice, say $z=w=\infty$ then $\operatorname{crt}_{\rho}(x, y, \infty, \infty)=(0: 1: 1)$.

The cross ratio triple is a more symmetric way to define the classical cross ratio $[., ., ., .]_{\rho}: Q \rightarrow[0, \infty]$ which we define as

$$
[x, y, z, w]_{\rho}:=\frac{\rho(x, z) \rho(y, w)}{\rho(x, y) \rho(z, w)} .
$$

It is not difficult to check that $\operatorname{crt}_{\rho}: Q \rightarrow \Sigma$ is continuous, where $Q$ and $\Sigma$ carry the obvious topologies induced by $(Z, \rho)$ and $\mathbb{R} P^{2}$. Thus, if $\left(x_{i}, y_{i}, z_{i}, w_{i}\right) \in Q$ for $i \in \mathbb{N}$ and assume $x_{i} \rightarrow x, \ldots, w_{i} \rightarrow w$, where $(x, y, z, w) \in Q$ then $\operatorname{crt}_{\rho}\left(x_{i}, y_{i}, z_{i}, w_{i}\right) \rightarrow \operatorname{crt}_{\rho}(x, y, z, w)$.

One can characterize convergence in $(Z, \rho)$ and the Cauchy-sequence property in terms of the cross ratio by the following Lemma (where we omit the proof) :

Lemma 2.2. Let $(Z, \rho)$ be an extended metric space. A sequence $\left(z_{i}\right) \subset Z$ converges to $z \in Z$, if and only if there are distinct points $a, b \in Z$, s.t. $\operatorname{crt}_{\rho}\left(z, z_{j}, a, b\right) \rightarrow(0: 1: 1)$.

A sequence $\left(z_{i}\right) \subset Z$ is a Cauchy-sequence in $(Z, \rho)$, if and only if there are distinct points $a, b \in Z$, s.t. $\operatorname{crt}_{\rho}\left(z_{i}, z_{j}, a, b\right) \rightarrow(0: 1: 1)$ for $i, j \rightarrow \infty$. 
A map $f:(Z, \rho) \rightarrow\left(Z^{\prime}, \rho^{\prime}\right)$ between two extended metric spaces is called Möbius, if $f$ is injective and for all admissible quadruples $(x, y, z, w)$ of $Z$,

$$
\operatorname{crt}_{\rho^{\prime}}(f(x), f(y), f(z), f(w))=\operatorname{crt}_{\rho}(x, y, z, w) .
$$

Now Lemma 2.2 implies that Möbius maps are continuos and Cauchysequences are mapped to Cauchy-sequences.

Two extended metric spaces $(Z, \rho)$ and $\left(Z^{\prime}, \rho^{\prime}\right)$ are Möbius equivalent, if there exists a bijective Möbius map $f: Z \rightarrow Z^{\prime}$. In this case also $f^{-1}$ is a Möbius map and $f$ is in particular a homeomorphism. Furthermore, by Lemma $2.2(Z, \rho)$ is complete if and only if $\left(Z^{\prime}, \rho^{\prime}\right)$ is.

We say that two extended metrics $\rho$ and $\rho^{\prime}$ on a set $Z$ are Möbius equivalent, if the identity map id : $(Z, \rho) \rightarrow\left(Z, \rho^{\prime}\right)$ is a Möbius map. Möbius equivalent metrics define the same topology on $Z$ and $(Z, \rho)$ is complete if and only $\left(Z, \rho^{\prime}\right)$ is.

A Möbius structure on a set $Z$ is a nonempty set $\mathcal{M}$ of extended metrics on $Z$, which are pairwise Möbius equivalent and which is maximal with respect to that property. Thus $\mathcal{M}$ is just an equivalence class of Möbius equivalent extended metrics on $Z$.

Given an extended metric $\rho$ on $Z$, we denote by $[\rho]$ the equivalence class of all Möbius equivalent extended metrics. Then $[\rho]$ is a Möbius structure on $Z$.

A Möbius space is a pair $(Z, \mathcal{M})$ of a set $Z$ with cardinality $|Z| \geq 3$ and a Möbius structure $\mathcal{M}$ on $Z$. If $(Z, \mathcal{M})$ is a Möbius space and $(x, y, z, w)$ an admissible quadruple, then the cross ratio triple is independent of the metric and hence $\operatorname{crt}(x, y, z, w):=\operatorname{crt}_{\mathcal{M}}(x, y, z, w):=\operatorname{crt}_{\rho}(x, y, z, w)$ is well defined, where $\rho \in \mathcal{M}$.

A Möbius space $(Z, \mathcal{M})$ is a topological space, since $\rho, \rho^{\prime} \in \mathcal{M}$ define the same topology on $Z$. A Möbius space is complete, if $(Z, \rho)$ is complete for $\rho \in \mathcal{M}$.

A map $f:(Z, \mathcal{M}) \rightarrow\left(Z^{\prime}, \mathcal{M}^{\prime}\right)$ between two Möbius spaces is called Möbius, if it is injective and preserves the cross ratio triple. The spaces are Möbius equivalent if there exists a bijective Möbius map between them.

Similar as for metric spaces, one can define the completion of a Möbius space $(Z, \mathcal{M})$. Define the set $\bar{Z}$ to be the set of equivalence classes of Cauchysequences, where two Cauchy-sequences $\left(x_{i}\right)$ and $\left(y_{i}\right)$ are called equivalent, if $\operatorname{crt}\left(x_{i}, y_{i}, a, b\right) \rightarrow(0: 1: 1)$ for some distinct $a, b$. If $\rho \in \mathcal{M}$ then define an extended metric $\bar{\rho}$ on $\bar{Z}$ by $\bar{\rho}\left(\left(x_{i}\right),\left(y_{i}\right)\right):=\lim \rho\left(x_{i}, y_{i}\right)$, here $\left(x_{i}\right)$ and $\left(y_{i}\right)$ are nonequivalent Cauchy-sequences. The completion of $(Z, \mathcal{M})$ is unique up to Möbius equivalence.

\subsection{Ultrametric Möbius structures}

A point $(a: b: c) \in \Sigma \subset \mathbb{R} P^{2}, a, b, c \geq 0$ is called ultrametric, if the two largest of the numbers $a, b, c$ coincide. The set of ultrametric points in $\Sigma$ 
is $Y$-shaped and consists out of the three affine segments from the central point $(1: 1: 1)$ to the three points $(0: 1: 1),(1: 0: 1),(1: 1: 0)$.

A Möbius space $(Z, \mathcal{M})$ is called ultrametric, if for all admissible quadruples $(x, y, z, w)$ the cross ratio triple $\operatorname{crt}(x, y, z, w)$ is ultrametric.

As usual, one calls an extended metric $\rho$ on a set $Z$ ultrametric, if for distinct $x, y, z \in Z_{\omega}$ the point $(\rho(x, y): \rho(x, z): \rho(y, z))$ is ultrametric.

If $(Z, \rho)$ is an extended ultrametric space and $\mathcal{M}=[\rho]$, then $(Z, \mathcal{M})$ is an ultrametric Möbius space. This follows e.g. from [BS] Lemma 5.1.2. On the other side, if $(Z, \mathcal{M})$ is ultrametric, then not necessarily all $\rho \in \mathcal{M}$ are ultrametrics. Consider for example the set $Z=\{x, y, z, w\}$ and let $\rho(x, y)=$ $t, \rho(z, w)=1 / t, \rho(x, z)=s, \rho(y, w)=1 / s, \rho(x, w)=r, \rho(y, z)=1 / r$, where $t, s, r$ are numbers close to one. The only relevant cross ratio triple is $\operatorname{crt}_{\rho}(x, y, z, w)=(1: 1: 1)$ which is ultrametric. All these metrics are Möbius equivalent but only for $s=t=r=1$ this is an ultrametric.

However in two cases one obtains ultrametrics:

Lemma 2.3. Let $(Z, \mathcal{M})$ be an ultrametric Möbius space and $\rho \in \mathcal{M}$ such that a remote point $\omega \in \Omega(\rho)$ exists. Then $\rho$ is an ultrametric.

Proof. Consider without loss of generality $x, y, z \in Z_{\omega}$. Then

$$
\operatorname{crt}_{\rho}(x, y, z, \omega)=(\rho(x, y): \rho(x, z): \rho(y, z)) .
$$

As $\operatorname{crt}_{\rho}$ is ultrametric it follows that $\rho$ is an ultrametric.

A metric $\rho$ on $Z$ is called antipodal diameter 1 metric, if $\operatorname{diam}(Z, \rho)=1$ and every $z \in Z$ has an antipodal point $z^{\prime} \in Z$, i.e. $\rho\left(z, z^{\prime}\right)=1$.

Lemma 2.4. Let $(Z, \mathcal{M})$ be an ultrametric Möbius space and $\rho \in \mathcal{M}$ an antipodal diameter 1 metric, then $\rho$ is an ultrametric.

Proof. Assume the contrary, then there are $x, y, z \in Z$ such that $\rho(x, y)>$ $\rho(x, z) \geq \rho(y, z)$. Let $z^{\prime} \in Z$ with $\rho\left(z, z^{\prime}\right)=1$. Then

$$
\rho(x, y) \rho\left(z, z^{\prime}\right)>\rho(x, z) \rho\left(y, z^{\prime}\right) \wedge \quad \rho(x, y) \rho\left(z, z^{\prime}\right)>\rho(y, z) \rho\left(x, z^{\prime}\right),
$$

which contradicts the fact that the cross ratio triple $\operatorname{crt}\left(x, y, z, z^{\prime}\right)$ is ultrametric.

An ultrametric Möbius space is in ptolemaic in the sense of [FS], which implies:

Lemma 2.5. Let $(Z, \mathcal{M})$ be an ultrametric Möbius space and let $\omega \in Z$, then there exists $\rho \in \mathcal{M}$, such that $\Omega(\rho)=\{\omega\}$. 


\section{Trees}

For this paper a tree is a metric space $(X, d)$ with the following two properties:

1. $X$ is uniquely geodesic: if $x, y \in X$, then there exists a unique isometric map $c:[0, \ell] \rightarrow X, \ell=d(x, y)$ with $c(0)=x$ and $c(\ell)=y$. The image of $c$ is denoted by $[x, y]$ and called the (geodesic) segment between $x$ and $y$. The points $x$ and $y$ are called the endpoints of $[x, y]$. Note that $[x, y]=[y, x]$.

2. If two geodesic segments $[x, y]$ and $[y, z]$ have only one point in common, which is an endpoint of each segment, then their union is also a segment; i.e.: if $[x, y] \cap[y, z]=\{y\}$ then $[x, z]=[x, y] \cup[y, z]$.

For the distance in $X$ we also use the notation $|x y|:=d(x, y)$.

A tree is called geodesically complete, if every nontrivial segment is contained in a geodesic line, i.e. if every isometric map $[0, \ell] \rightarrow X, \ell>0$ extends to an isometric map $\mathbb{R} \rightarrow X$.

Trees are CAT $(-1)$ spaces in the sense of comparison geometry (see e.g. $[\mathrm{BS}])$ and are in particular geodesic Gromov hyperbolic spaces. Actually they are 0 -hyperbolic and $\operatorname{CAT}(\kappa)$ for all $\kappa \in \mathbb{R}$. We now collect some well known facts about trees.

If $x, y, z \in X$ are three points, then there exists a unique point $w:=$ $\operatorname{Trip}(x, y, z) \in X$ with $\{w\}=[x, y] \cap[y, z] \cap[x, z]$. The point $w$ is called the tripod of $x, y, z$.

We have $|x w|=(z \mid y)_{x},|y w|=(x \mid z)_{y}$ and $|z w|=(x \mid y)_{z}$, where we use the so called Gromov product $(x \mid y)_{z}:=\frac{1}{2}(|z x|+|z y|-|x y|)$.

To define the boundary at infinity we call a sequence $\left(x_{i}\right)$ in $X$ a Gromov sequence, if for some basepoint $o \in X,\left(x_{i} \mid x_{j}\right)_{o} \rightarrow \infty$ for $i, j \rightarrow \infty$. Two Gromov sequences $\left(x_{i}\right),\left(y_{i}\right)$ are called equivalent, if $\left(x_{i} \mid y_{i}\right)_{o} \rightarrow \infty$. The equivalence classes are the points of the boundary $\partial_{\infty} X$.

We call a tree $X$ non-elementary, if the cardinality $\left|\partial_{\infty} X\right| \geq 3$. Thus (up to isometry) the only nontrivial elementary geodesically complete tree is the euclidean line $\mathbb{R}$.

Given a point $a \in \partial_{\infty} X$ and a point $x \in X$, there exists a unique geodesic ray (i.e. a unique isometric map) $c:[0, \infty) \rightarrow X$, such that $c(0)=x$ and $c(\infty)=a$ (which means that the sequence $c(i), i \in \mathbb{N}$, represents $a$ ). We denote the image of this ray as $[x, a)$.

Given two distinct points $a, b \in \partial_{\infty} X$ and $x \in X$, there exists a unique point $u \in X$ such that $[x, a) \cap[x, b)=[x, u]$. Then $(a, b):=[u, a) \cup[u, b)$ is the image of an isometric embedding $\mathbb{R} \rightarrow X$ and called the line between $a$ and $b$. If $\left(a_{i}\right),\left(b_{i}\right)$ are sequences in $X$ with $a_{i} \rightarrow a$ and $b_{i} \rightarrow b$, then $\left(a_{i} \mid b_{i}\right)_{x}=|x u|$ for $i$ sufficiently large. We define $(a \mid b)_{x}:=\lim \left(a_{i} \mid b_{i}\right)_{x}=|x u|$. 
For $x, y \in X$ and $a \in \partial_{\infty} X$ we also define $(a \mid x)_{y}:=\lim \left(a_{i} \mid x\right)_{y}$, where $\left(a_{i}\right)$ is a sequence in $X$ converging to $a$.

If $a \in \partial_{\infty} X$, the Busemann function $B_{a}: X \times X \rightarrow \mathbb{R}$ is defined as

$$
B_{a}(x, y)=\lim \left(\left|x a_{i}\right|-\left|y a_{i}\right|\right)=(a \mid y)_{x}-(a \mid x)_{y}
$$

where $\left(a_{i}\right)$ is a sequence converging to $a$. All these limits are well defined.

For $x \in X$ and $a, b \in \partial_{\infty} X$ let $\rho_{x}(a, b):=e^{-(a \mid b)_{x}}$. The $\rho_{x}$ defines the Bourdon metric (compare [Bou] ) on $\partial_{\infty} X$ with basepoint $x \in X$. This is an ultrametric. Since $(a \mid b)_{x}-(a \mid b)_{y}=\frac{1}{2}\left(B_{a}(x, y)+B_{b}(x, y)\right)$, we have

$$
\rho_{y}(a, b)=\lambda(a) \lambda(b) \rho_{x}(a, b)
$$

where $\lambda=\lambda_{x, y}: \partial_{\infty} X \rightarrow \mathbb{R}$ is $\lambda(a)=e^{\frac{1}{2} B_{a}(x, y)}$.

In particular the metrics $\rho_{x}$ and $\rho_{y}$ are Möbius equivalent and they define (in the case that $X$ is nonelementary) the same Möbius structure on $\partial_{\infty} X$.

We denote this Möbius structure by $\mathcal{M}_{X}$ and call it the canonical Möbius structure on $X$. This Möbius structure is ultrametric.

For $\omega \in \partial_{\infty} X$ and $o \in X$ we define for $a, b \in \partial_{\infty} X \backslash\{\omega\}$

$$
(a \mid b)_{\omega, o}:=(a \mid b)_{o}-(a \mid \omega)_{o}-(b \mid \omega)_{o} .
$$

Then

$$
\rho_{\omega, o}(a, b):=e^{-(a \mid b)_{\omega, o}}=\frac{\rho_{o}(a, b)}{\rho_{o}(a, \omega) \rho_{o}(b, \omega)}
$$

defines a metric on $\partial_{\infty} X \backslash\{\omega\}$ which can be considered as an extended metric on $\partial_{\infty} X$ with remote point $\omega$. This metric is also contained in $\mathcal{M}_{X}$.

\section{Geodesically complete trees versus ultrametric Möbius spaces}

Theorem 4.1. Let $(Z, \mathcal{M})$ be a complete ultrametric Möbius space. Then there is a nonelementary geodesically complete tree $X$, such that $\left(\partial_{\infty} X, \mathcal{M}_{X}\right)$ is Möbius equivalent to $(Z, \mathcal{M})$.

Proof. By Lemma 2.5 we know that for an ultrametric Möbius structure $(Z, \mathcal{M})$ there is $\rho \in \mathcal{M}$ such that $\Omega(\rho)=\{\omega\}$. By Lemma $2.3 \rho$ is defines an ultrametric on $Z_{\omega}$. We define the space

$$
X:=\left(Z_{\omega} \times \mathbb{R}\right) / \sim
$$

where

$$
\left(z_{1}, t_{1}\right) \sim\left(z_{2}, t_{2}\right) \Longleftrightarrow t_{1}=t_{2} \wedge t_{1} \leq-\ln \rho\left(z_{1}, z_{2}\right)
$$


for $z_{1}, z_{2} \in Z_{\omega}$ and $t_{1}, t_{2} \in \mathbb{R}$. Since $\rho$ is an ultrametric $\sim$ is an equivalence relation. The space $X$ will be the "filling" of $Z$. Therefore we define

$$
d\left(\left[z_{1}, t_{1}\right],\left[z_{2}, t_{2}\right]\right):=t_{1}+t_{2}-2 \min \left(t_{1}, t_{2},-\ln \rho\left(z_{1}, z_{2}\right)\right) .
$$

Similar to section 6 of $[\mathrm{Hu}]$ one can show that $d$ is a well defined metric. We first show that $(X, d)$ has a natural bicombing, i.e. given $\left[z_{1}, t_{1}\right],\left[z_{2}, t_{2}\right] \in$ $X$, there is a natural geodesic between these points, which can be described as follows:

Assume w.l.o.g. $t_{1}<t_{2}$. If $t_{1} \leq-\ln \rho\left(z_{1}, z_{2}\right)$, the natural geodesic joining $\left[z_{1}, t_{1}\right]$ to $\left[z_{2}, t_{2}\right]$ is $\gamma:\left[t_{1}, t_{2}\right] \rightarrow X, \gamma(s):=\left[z_{2}, s\right]$. If $t_{1}>-\ln \rho\left(z_{1}, z_{2}\right)$, let $a:=t_{1}+\ln \rho\left(z_{1}, z_{2}\right)+t_{2}+\ln \rho\left(z_{1}, z_{2}\right)$. We define the natural geodesic joining $\left[z_{1}, t_{1}\right]$ to $\left[z_{2}, t_{2}\right]$ to be

$$
\gamma:[0, a] \rightarrow X \quad \gamma(s):= \begin{cases}{\left[z_{1}, t_{1}-s\right]} & s \leq t_{1}+\ln \rho\left(z_{1}, z_{2}\right) \\ {\left[z_{2},-\ln \rho\left(z_{1}, z_{2}\right)+s\right]} & s>t_{1}+\ln \rho\left(z_{1}, z_{2}\right)\end{cases}
$$

It is straight forward to check that $\gamma$ is a geodesic with $\gamma(0)=\left[z_{1}, t_{1}\right]$ and $\gamma(a)=\left[z_{2}, t_{2}\right]$. The bicombing implies in particular that $X$ is geodesic. For any three points $\left[z_{1}, t_{1}\right],\left[z_{2}, t_{2}\right],\left[z_{3}, t_{3}\right] \in X$ there is a geodesic triangle formed by natural geodesics. Using the ultrametric property of $\rho$ we can assume w.l.o.g. $\rho\left(z_{1}, z_{2}\right) \leq \rho\left(z_{1}, z_{3}\right)=\rho\left(z_{2}, z_{3}\right)$. By definition of the natural geodesics it follows that this geodesic triangle is 0-thin in the sense of Gromov and the tripod is $\left[z_{1},-\ln \rho\left(z_{1}, z_{2}\right)\right]$. In particular for any three points $x, y, z \in X$, there exists a tripod $u$ which is contained in all natural geodesics between the three points. Thus the equality $d(x, z)+d(z, y)=d(x, y)$ holds only if $z=u$ and hence $z$ lies on the natural geodesic from $x$ to $y$. This implies that the natural geodesics are (up to parametrization) the unique geodesics. Thus $X$ is uniquely geodesics and satisfies the first defining property of a tree. By definition of the natural geodesics it is easy to check that also the second defining property of a tree is satisfied.

In order to prove that $X$ is geodesically complete, we will show that any two points lie on a geodesic line. As both types of natural geodesics can be extended to bi-infinite lines, namely $\gamma$ is either of the form $\gamma(t)=[z, t]$ for some $z \in Z_{\omega}$ or $\gamma(t)=\left[z_{1},-t\right]$ for $t<-\ln \rho\left(z_{1}, z_{2}\right)$ and $\gamma(t)=\left[z_{2}, t\right]$ for $t \geq-\ln \rho\left(z_{1}, z_{2}\right), X$ is geodesically complete.

We will show that the geodesic boundary of $X$ equals $Z$. We define a map $\iota: Z \rightarrow \partial_{\infty} X$ in the following way: For $z \in Z_{\omega}$ let $\iota(z):=\gamma_{z}(\infty)$ where $\gamma_{z}(t):=[z, t]$. Furthermore $\iota(\omega):=\bar{\gamma}_{z}(\infty)$ where $\bar{\gamma}_{z}(t):=[z,-t]$ and $z \in Z_{\omega}$ is arbitrary. Note that for different $z \in Z_{\omega}$ the rays $\bar{\gamma}_{z}$ are equivalent in terms of the geodesic boundary, as $\left[z_{1},-t\right]=\left[z_{2},-t\right]$ for $-t \leq-\ln \rho\left(z_{1}, z_{2}\right)$. It is clear that the map $\iota$ is injective.

To show surjectivity let $v^{\prime} \in \partial_{\infty} X \backslash\{\iota(\omega)\}$ be given. Let $\gamma:[0, \infty) \rightarrow X$ be the unit speed ray with $\gamma(0)=[u, 0]$, where $u \in Z_{\omega}$ is some basepoint, and 
$\gamma(\infty)=v^{\prime}$. We know that two points can be joined by the unique natural geodesic as described above. Since $v^{\prime} \neq \iota(\omega)$ we see that there is $s_{0} \in[0, \infty)$, such that $\gamma\left(s_{0}\right)=\left[z_{s_{0}}, 0\right]$ and for $s>s_{0}$ it is $\gamma(s)=\left[z_{s}, s-s_{0}\right]$ for some points $z_{s} \in Z_{\omega}$. Consider now numbers $s^{\prime}>s>s_{0}$. We have $d\left(\gamma\left(s^{\prime}\right), \gamma(s)\right)=s^{\prime}-s$ as well as $d\left(\gamma\left(s^{\prime}\right), \gamma(s)\right)=s^{\prime}+s-2 s_{0}-\min \left\{s-s_{0},-\ln \rho\left(z_{s}, z_{s^{\prime}}\right)\right\}$. Hence $-\ln \rho\left(z_{s}, z_{s^{\prime}}\right) \geq s-s_{0}$. By completeness of $(Z, \rho)$ this implies that the points $z_{s}$ converge in $Z_{\omega}$ for $s \rightarrow \infty$. Let $z_{s} \rightarrow v \in Z_{\omega}$ for $s \rightarrow \infty$. Then $\left[z_{s}, t\right]=[v, t]$ for $t$ sufficiently large. Thus $\gamma(s)=\left[v, s-s_{0}\right]$ for $s$ large which implies that $\iota(v)=v^{\prime}$.

Finally we show that the Möbius structures coincide. Choose a basepoint $u \in Z_{\omega}$. We actually show that $\iota:(Z, \rho) \rightarrow\left(\partial_{\infty} X, \rho_{\omega^{\prime},[u, 0]}\right)$ is an isometry, where $\rho_{\omega^{\prime},[u, 0]}=e^{-(. \mid)_{\omega^{\prime},[u, 0]}}$ is the metric in the canonical Möbius structure of $\partial_{\infty} X$ with remote point $\omega^{\prime}=\iota(\omega)$.

For $a, b \in Z_{\omega}$ we compute, where we use $a^{\prime}=\iota(a), b^{\prime}=\iota(b)$,

$$
\begin{aligned}
\left(a^{\prime} \mid b^{\prime}\right)_{\omega^{\prime},[u, 0]} & =\left(a^{\prime} \mid b^{\prime}\right)_{[u, 0]}-\left(a^{\prime} \mid \omega^{\prime}\right)_{[u, 0]}-\left(b^{\prime} \mid \omega^{\prime}\right)_{[u, 0]} \\
& =\lim _{t \rightarrow \infty}\left(([a, t] \mid[b, t])_{[u, 0]}-([a, t] \mid[u,-t])_{[u, 0]}-([b, t] \mid[u,-t])_{[u, 0]}\right) \\
& =\lim _{t \rightarrow \infty} \frac{1}{2}(2 t-d([a, t],[b, t])) \\
& =\lim _{t \rightarrow \infty} \frac{1}{2}(2 t-(2 t-2 \min \{t,-\ln \rho(a, b)\})) \\
& =-\ln \rho(a, b)
\end{aligned}
$$

which implies $\rho_{\omega^{\prime},[u, 0]}\left(a^{\prime}, b^{\prime}\right)=\rho(a, b)$.

Lemma 4.2. Let $(X, d)$ be a tree and let $a, b, c_{1}, c_{2} \in \partial_{\infty} X$ points, such that $a, b, c_{1}$ and $a, b, c_{2}$ are distinct. Let $u_{i}=\operatorname{Trip}\left(a, b, c_{i}\right)$, then

$$
\left|u_{1} u_{2}\right|= \pm \ln \left(\left[a, c_{1}, c_{2}, b\right]\right),
$$

while we have a positive sign if $u_{2} \in\left[u_{1}, b\right)$ and a negative if $u_{1} \in\left[u_{2}, a\right)$.

Proof. Let $o \in(a, b)$ s.t. $u_{1}, u_{2} \in[o, b)$ and $\rho_{o}$ denotes the Bourdon metric with some basepoint $o \in X$. Let $\mu_{1}=\left|o u_{1}\right|$ and $\mu_{2}=\left|o u_{2}\right|$, then it is $\rho_{o}\left(a, c_{1}\right)=1, \rho_{o}\left(a, c_{2}\right)=1, \rho_{o}\left(b, c_{1}\right)=e^{-\mu_{1}}, \rho_{o}\left(b, c_{2}\right)=e^{-\mu_{2}}$ and hence

$$
\begin{aligned}
& {\left[a, c_{1}, c_{2}, b\right]=\frac{\rho_{o}\left(a, c_{2}\right) \rho_{o}\left(c_{1}, b\right)}{\rho_{o}\left(b, c_{2}\right) \rho_{o}\left(c_{1}, a\right)}=e^{-\mu_{1}+\mu_{2}} . } \\
\Longrightarrow & \ln \left(\left[a, c_{1}, c_{2}, b\right]\right)=\mu_{2}-\mu_{1} .
\end{aligned}
$$

If $\mu_{1} \leq \mu_{2}, \ln \left(\left[a, c_{1}, c_{2}, b\right]\right)=\left|u_{1} u_{2}\right|$ and if $\mu_{1} \geq \mu_{2}, \ln \left(\left[a, c_{1}, c_{2}, b\right]\right)=-\left|u_{1} u_{2}\right|$, which proves the claim. 
Theorem 4.3. Let $X$ be a nonelementary geodesically complete tree and let $X^{\prime}$ be an arbitrary tree with $\left|\partial_{\infty} X^{\prime}\right| \geq 3$. Then for any Moebius embedding $f: \partial_{\infty} X \rightarrow \partial_{\infty} X^{\prime}$ w.r.t. the natural Moebius structures, there exists a unique isometric embedding $F: X \rightarrow X^{\prime}$. s.t. the extension of $F$ to the boundary coincides with $f$.

If $f$ is in addition surjective and $X^{\prime}$ geodesically complete, then $F$ is an isometry.

Proof. First we define a map $F: X \rightarrow X^{\prime}$. For a given $x \in X$ there are, since $X$ is nonelementary and geodesically complete, points $a, b \in \partial_{\infty} X$, s.t. $x \in(a, b)$. Let $c \in \partial_{\infty} X \backslash\{a, b\}$ and $\gamma_{a, b, c}$ the unit speed geodesic with $\gamma(-\infty)=a, \gamma(\infty)=b$ and $\gamma(0)=\operatorname{Trip}(a, b, c)$. Then $x=\gamma\left(t_{x}\right)$ for $t_{x}= \pm|\operatorname{Trip}(a, b, c) x|$, while the sign is positive if $x \in[\operatorname{Trip}(a, b, c), b)$ and negative if $x \in(a, \operatorname{Trip}(a, b, c)]$. Let $a^{\prime}=f(a), b^{\prime}=b$ and $c^{\prime}=c$, we define $F_{a, b, c}(x):=\gamma_{a^{\prime}, b^{\prime}, c^{\prime}}^{\prime}\left(t_{x}\right)$ for $\gamma_{a^{\prime}, b^{\prime}, c^{\prime}}^{\prime}$ the unit speed geodesic with $\gamma^{\prime}(-\infty)=a^{\prime}$, $\gamma^{\prime}(\infty)=b^{\prime}$ and $\gamma^{\prime}(0)=\operatorname{Trip}\left(a^{\prime}, b^{\prime}, c^{\prime}\right)$. We have to show that $F_{a, b, c}$ is independent of different choices of $a, b$ and $c$. Let $a_{1}, b_{1}, c_{1}, a_{2}, b_{2}, c_{2} \in \partial_{\infty} X$, s.t. $x \in\left(a_{1}, b_{1}\right), x \in\left(a_{2}, b_{2}\right)$ and the three points $a_{i}, b_{i}, c_{i}$ are mutually distinct for $i=1,2$. In three steps we will show the following

Claim: $F_{a_{1}, b_{1}, c_{1}}(x)=F_{a_{2}, b_{2}, c_{2}}(x)$.

Step 1: $F_{a, b, c}(x)=F_{b, a, c}(x)$

By construction it follows $\gamma_{b, a, c}(-t)=\gamma_{a, b, c}(t)$, the absolute value of $t_{x}$ stays the same, but the sign of $t_{x}$ changes by interchanging $a$ and $b$ in the above construction. In particular this implies $F_{a, b, c}(x)=F_{b, a, c}(x)$.

Step 2: If $x \in(a, b) \cap\left(a, b^{\prime}\right)$ then $F_{\left(a, b, b^{\prime}\right)}(x)=F_{\left(a, b^{\prime}, b\right)}(x)$ and if $x \in$ $(a, b) \cap\left(a^{\prime}, b\right)$ then $F_{\left(a, b, a^{\prime}\right)}(x)=F_{\left(a^{\prime}, b, a\right)}(x)$

Note that $\gamma_{a, b, b^{\prime}}$ and $\gamma_{a, b^{\prime}, b}$ coincide on $(-\infty, 0]$ and $\gamma_{a, b, a^{\prime}}$ coincides with $\gamma_{a^{\prime}, b, a}$ on $[0, \infty)$.

Step 3: $F_{a, b, c_{1}}(x)=F_{a, b, c_{2}}(x)$ in the case that $c_{1}, c_{2} \in \partial_{\infty} X \backslash\{a, b\}$

Let $u_{i}=\operatorname{Trip}\left(a, b, c_{i}\right)$ and $t_{x}\left(a, b, c_{i}\right)= \pm\left|\operatorname{Trip}\left(a, b, c_{i}\right) x\right|$ be the signed distances as in the construction. By construction it's

$$
\begin{array}{lll}
t_{x}\left(a, b, c_{1}\right)=t_{x}\left(a, b, c_{2}\right)-\left|u_{1} u_{2}\right| & \text { if } & u_{2} \in\left[u_{1}, b\right) \\
t_{x}\left(a, b, c_{1}\right)=t_{x}\left(a, b, c_{2}\right)+\left|u_{1} u_{2}\right| & \text { if } & u_{1} \in\left[u_{2}, b\right) .
\end{array}
$$

Let $u_{i}^{\prime}=\operatorname{Trip}\left(a^{\prime}, b^{\prime}, c_{i}^{\prime}\right)$ and let $\pm\left|F_{a, b, c_{2}}(x) u_{1}^{\prime}\right|$ be the signed distance being positive if $F_{a, b, c_{2}}(x) \in\left[u_{1}^{\prime}, b^{\prime}\right)$ and negative if $u_{1}^{\prime} \in\left[F_{a, b, c_{2}}(x), b^{\prime}\right)$. Due to elementary arguments it follows

$$
\begin{array}{lll} 
\pm\left|F_{a, b, c_{2}}(x) u_{1}^{\prime}\right|=t_{x}\left(a, b, c_{2}\right)-\left|u_{1}^{\prime} u_{2}^{\prime}\right| & \text { if } & u_{2}^{\prime} \in\left[u_{1}^{\prime}, b^{\prime}\right) \\
\pm\left|F_{a, b, c_{2}}(x) u_{1}^{\prime}\right|=t_{x}\left(a, b, c_{2}\right)+\left|u_{1}^{\prime} u_{2}^{\prime}\right| & \text { if } & u_{1}^{\prime} \in\left[u_{2}^{\prime}, b^{\prime}\right) .
\end{array}
$$

We have $\left[a, c_{1}, c_{2}, b\right]=\left[a^{\prime}, c_{1}^{\prime}, c_{2}^{\prime}, b^{\prime}\right]$, as $f$ is a Möbius map. Using this and Lemma 4.2, it follows that $\pm\left|F_{a, b, c_{2}}(x) u_{1}^{\prime}\right|=t_{x}\left(a, b, c_{1}\right)$. By construction $t_{x}\left(a, b, c_{1}\right)= \pm\left|F_{a, b, c_{1}}(x) u_{1}^{\prime}\right|$. Thus $F_{a, b, c_{1}}(x)$ and $F_{a, b, c_{2}}(x)$ lie on the 
geodesic $\left(a^{\prime}, b^{\prime}\right)$ with the same signed distance to the point $u_{1}^{\prime}$, hence it follows $F_{a, b, c_{1}}(x)=F_{a, b, c_{2}}(x)$.

Using step 1 and step 3 the claim follows in the case that $\left\{a_{1}, b_{1}\right\}=$ $\left\{a_{2}, b_{2}\right\}$. Thus (by eventually interchanging $a_{2}$ and $b_{2}$ ) we can assume that $b_{1} \neq b_{2}$. Consider first the case $a_{1}=a_{2}$. Using step 2 and step 3 we get

$$
F_{a_{1}, b_{1}, c_{1}}(x)=F_{a_{1}, b_{1}, b_{2}}(x)=F_{a, b_{2}, b_{1}}(x)=F_{a_{1}, b_{2}, c_{2}}(x)
$$

and hence the claim. Let us now assume that $a_{1}, a_{2}, b_{1}, b_{2}$ are all distinct. Let $u=\operatorname{Trip}\left(a_{1}, b_{1}, a_{2}\right)$ and $v=\operatorname{Trip}\left(a_{1}, b_{1}, b_{2}\right)$. By eventually interchanging $a_{2}$ and $b_{2}$, we can assume that the order of the points on the line $\left(a_{1}, b_{1}\right)$ are $a_{1}<u<v<b_{1}$. Note that then $x \in\left(a_{i}, b_{j}\right)$ for every choice of $i, j$. Using the various equalities we obtain:

$$
\begin{aligned}
& F_{a_{1}, b_{1}, c_{1}}(x)=F_{a_{1}, b_{1}, b_{2}}(x)=F_{a_{1}, b_{2}, b_{1}}(x)=F_{b_{2}, a_{1}, b_{1}}(x) \\
= & F_{b_{2}, a_{1}, a_{2}}(x)=F_{b_{2}, a_{2}, a_{1}}(x)=F_{a_{2}, b_{2}, a_{1}}(x)=F_{a_{2}, b_{2}, c_{2}}(x)
\end{aligned}
$$

In particular we have a well defined map $F: X \rightarrow X^{\prime}$ by $F(x):=$ $F_{a, b, c}(x), a, b, c \in \partial_{\infty} X$ distinct and $x \in(a, b)$.

We show that $F$ is an isometry. Let $x, y \in X$ be arbitrary, due to geodesically completeness there are $a, b \in \partial_{\infty} X$, s.t. $x, y \in(a, b)$. As the distance $|x y|$ is determined by the signed distances of $x$ and $y$ to $\operatorname{Trip}(a, b, c)$ for some $c \in \partial_{\infty} X \backslash\{a, b\}$ and $F(x), F(y)$ have the same signed distances to $\operatorname{Trip}\left(a^{\prime}, b^{\prime}, c^{\prime}\right)$ by construction, it follows that $|x y|=|F(x) F(y)|$.

It follows immediately from the construction that for distinct points $a, b \in$ $\partial_{\infty} X, F$ maps the line $(a, b)$ to the line $(f(a), f(b)$. Thus restricted to the boundary $F$ equals $f$.

We show that such an $F$ is unique. Any isometry $\hat{F}: X \rightarrow X^{\prime}$ with $\hat{F}_{\mid \partial_{\infty} X}=f$ maps the line $(a, b)$ for $a, b \in \partial_{\infty} X$ to the line $(f(a), f(b))$. Let $\hat{F}$ and $F$ be such isometries, then they differ on $(a, b)$ by a translation. If we take $c \in \partial_{\infty} X \backslash\{a, b\}$ it is $\operatorname{Trip}(a, b, c)=(a, b) \cap(b, c) \cap(a, c)$, which shows that $F(\operatorname{Trip}(a, b, c))=\operatorname{Trip}(f(a), f(b), f(c))=\hat{F}(\operatorname{Trip}(a, b, c))$. In particular $F_{\mid(a, b)}=\hat{F}_{\mid(a, b)}$. But as the geodesic $(a, b)$ was arbitrary and $X$ is geodesically complete it follows $F=\hat{F}$.

The last step is to show that $F$ is surjective if $f$ is surjective and $X^{\prime}$ is geodesically complete. Let $x^{\prime} \in X^{\prime}$ be arbitrary, then there is by geodesically completeness $a^{\prime}, b^{\prime} \in \partial_{\infty} X^{\prime}$, s.t. $x^{\prime} \in\left(a^{\prime}, b^{\prime}\right)$. Let $c^{\prime} \in \partial_{\infty} X \backslash\left\{a^{\prime}, b^{\prime}\right\}$ then $x^{\prime}$ is determined by the signed distance to Trip $\left(a^{\prime}, b^{\prime}, c^{\prime}\right)$. In particular this implies that the point $x \in\left(f^{-1}\left(a^{\prime}\right), f^{-1}\left(b^{\prime}\right)\right)$ with the same signed distance to $\operatorname{Trip}\left(f^{-1}\left(a^{\prime}\right), f^{-1}\left(b^{\prime}\right), f^{-1}\left(c^{\prime}\right)\right)$ is mapped to $x^{\prime}$ by construction. We note that $f^{-1}$ exists, as $f$ is as a Möbius map injective and assumed to be surjective.

Corollary 4.4. The tree constructed in theorem 4.1 is unique up to isometry. 
Proof. Let $X, X^{\prime}$ be two such trees. As the boundaries with the natural Möbius structures both equal $(Z, \mathcal{M})$ (up to Möbius isomorphism), there is a Möbius isomorphism $f: \partial_{\infty} X \rightarrow \partial_{\infty} X^{\prime}$. If we apply the theorem above the result follows.

\section{$5 \quad$ Antipodal diameter 1 metrics}

In this section we recall some facts from [Bi] and give a slight generalization of one of its result.

Let $(Z, \mathcal{M})$ be an arbitrary Möbius space. By $\mathcal{M}_{1}^{a}$ we denote the subset of antipodal, diameter 1 metrics. The set $\mathcal{M}_{1}^{a}$ may be empty.

Biswas has showed in [Bi] that there is a natural metric on $\mathcal{M}_{1}^{a}$. We note that he assumed $Z$ to be compact, but for the definition of this metric that is not necessary. We recall some of the notation and the definition of the metric.

Let $(Z, \mathcal{M})$ be an arbitrary Möbius structure and $\mathcal{M}_{1}^{a}$ the subset of antipodal diameter one metrics. For $\rho_{1}, \rho_{2} \in \mathcal{M}_{1}^{a}$ and $\xi \in Z$ we define

$$
\frac{d \rho_{1}}{d \rho_{2}}(\xi):=\frac{\rho_{1}\left(\eta, \eta^{\prime}\right)}{\rho_{1}(\xi, \eta) \rho_{1}\left(\xi, \eta^{\prime}\right)} \frac{\rho_{2}(\xi, \eta) \rho_{2}\left(\xi, \eta^{\prime}\right)}{\rho_{2}\left(\eta, \eta^{\prime}\right)},
$$

with $\eta, \eta^{\prime} \in Z \backslash\{\xi\}$ arbitrary but distinct. One can show that this definition is independent of the choice of $\eta, \eta^{\prime} \in Z \backslash\{\xi\}$. Similar as in [Bi] one can show that

$$
d_{\mathcal{M}_{1}^{a}}\left(\rho_{1}, \rho_{2}\right):=\sup _{\zeta \in Z} \ln \frac{d \rho_{1}}{d \rho_{2}}(\zeta)
$$

is a metric on $\mathcal{M}_{1}^{a}$. Furthermore he shows that for a CAT(-1) space $X$ and $\rho_{x}, \rho_{y}$ Bourdon metrics, we have $\frac{d \rho_{y}}{d \rho_{x}}=\lambda_{x, y}^{2}$. By the definition of $\lambda$ it follows $\ln \frac{d \rho_{y}}{d \rho_{x}}(\zeta)=B_{\zeta}(x, y)$

Theorem 5.1. Let $(Z, \mathcal{M})$ be a ultrametric Möbius space, and let $(X, d)$ be a geodesically complete tree such that $\left(\partial_{\infty} X, \mathcal{M}_{X}\right)=(Z, \mathcal{M})$. Let $i$ : $(X, d) \rightarrow\left(\mathcal{M}_{1}^{a}, d_{\mathcal{M}_{1}^{a}}\right)$ be the map sending $x$ to the Bourdon metric $\rho_{x}$, then $i$ is an isometry.

Proof. The first step is to show that $i$ is bijective. First observe that the geodesically completeness of $(X, d)$ implies that for every $x \in X$, the metric $\rho_{x}$ is antipodal and of diameter 1 . This implies that the image $i$ lies in $\mathcal{M}_{1}^{a}$. As $Z$ has cardinality greater than two, it easily follows that $\rho_{x} \neq \rho_{y}$ for $x \neq y$, which means that $i$ is injective.

We show that $i$ is surjective. Let $\rho \in \mathcal{M}_{1}^{a}$ be given. Let $a, b \in \partial_{\infty} X$, s.t. $\rho(a, b)=1$ and $c \in \partial_{\infty} X$ arbitrary, but distinct from $a$ and $b$. By lemma 2.4 $\rho$ is an ultrametic, therefore $\rho(a, c)=1$ or $\rho(b, c)=1$. W.l.o.g. we assume 
$\rho(a, c)=1$. Let $x$ be in $[\operatorname{Trip}(a, b, c), a)$, s.t. $|x \operatorname{Trip}(a, b, c)|=-\ln (\rho(b, c))$, then $\left(\rho_{x}\right)_{\mid\{a, b, c\}}=\rho_{\mid\{a, b, c\}}$. For $d \in \partial_{\infty} X \backslash\{a, b, c\}$ and $\mu:=\rho(c, b)=\rho_{x}(c, b)$, we have by the Möbius equivalence of $\rho$ and $\rho_{x}$

$$
\begin{aligned}
& (\rho(c, d): \rho(b, d): \mu \rho(a, d))=\operatorname{crt}_{\rho}(c, d, a, b)=\operatorname{crt}_{\rho_{x}}(c, d, a, b) \\
= & \left(\rho_{x}(c, d): \rho_{x}(b, d): \mu \rho_{x}(a, d)\right) .
\end{aligned}
$$

Thus there is a factor $\nu>0$ such that we have the vector equality

$$
\nu(\rho(c, d), \rho(b, d), \mu \rho(a, d))=\left(\rho_{x}(c, d), \rho_{x}(b, d), \mu \rho_{x}(a, d)\right) .
$$

We will show that $\nu=1$. As $\rho$ and $\rho_{x}$ are ultrametrics we have $\rho(b, d)=1$ or $\rho(a, d)=1$, as well as $\rho_{x}(b, d)=1$ or $\rho_{x}(a, d)=1$. If $\rho_{x}(a, d)=1 \wedge \rho(a, d)=1$ then $\nu=1$ and in the same way $\rho_{x}(b, d)=1 \wedge \rho(b, d)=1$ implies $\nu=1$. One easily sees that in the remaining cases

$$
\rho_{x}(a, d)=1 \wedge \rho(a, d)<1 \wedge \rho(b, d)=1 \wedge \rho_{x}(b, d)<1
$$

and

$$
\rho_{x}(a, d)<1 \wedge \rho(a, d)=1 \wedge \rho(b, d)<1 \wedge \rho_{x}(b, d)=1
$$

the vector equality from above can not be satisfied. Thus $\nu=1$, which implies that $\left(\rho_{x}\right)_{\mid\{a, b, c, d\}}=\rho_{\mid\{a, b, c, d\}}$. In particular this means that the construction of $x$ is independent of the choice $c$ or $d$. Furthermore it follows that for arbitrary $c, d \in \partial_{\infty} X$ we have $\rho_{x}(c, d)=\rho(c, d)$. Hence $\rho$ is the Bourdon metric $\rho_{x}$.

Finally we have to show $d(x, y)=d_{\mathcal{M}_{1}^{a}}\left(\rho_{x}, \rho_{y}\right)$. For given points $x, y \in X$ we can extend the geodesic segment $[x, y]$ to a geodesic ray $[x, \xi)$ where $\xi \in \partial_{\infty} X$. Thus $d(x, y)=B_{\xi}(x, y)$. Since $B_{\zeta}(x, y) \leq d(x, y)$ for all $\zeta \in \partial_{\infty} X$ we have

$$
d_{\mathcal{M}_{1}^{a}}\left(\rho_{x}, \rho_{y}\right)=\sup _{\zeta \in \partial_{\infty} X} \ln \frac{d \rho_{y}}{d \rho_{x}}(\zeta)=\sup _{\zeta \in \partial_{\infty} X} B_{\zeta}(x, y)=B_{\xi}(x, y)=d(x, y) .
$$

\section{References}

[Bi] K. Biswas, On Moebius and Conformal Maps Between Boundaries of CAT(-1) Spaces, arXiv:1203.6212v3 [math.DS] (2013)

[Bou] M. Bourdon, Structure conforme au bord et flot géodésique d'un CAT(-1)-espace, L'Einseignement Mathématique 41 (1995) 63-102

[BS] S. Buyalo, V. Schroeder, Elements of asymptotic geometry, EMS Monographs in Mathematics, 2007, 209 pages. 
[FS] T. Foertsch, V. Schroeder, Hyperbolicity, CAT(-1)-spaces and Ptolemy inequality, Math. Ann. 350 (2011), no. 2, 339-356.

[Hu] B. Hughes, Trees and ultrametric spaces: a categorical equivalence, Advances in Mathematics 189 (2004) 148 - 191

Jonas Beyrer

Viktor Schroeder

Institut für Mathematik

Universität Zürich

Winterthurer Strasse 190

CH-8057 Zürich, Switzerland

jonas . beyrer@math.uzh.ch

viktor.schroeder@math.uzh.ch 